\title{
A RHOse by any other name: a comparative analysis of animal and plant Rho GTPases
}

\author{
Tore Brembu ${ }^{1,2}$, Per Winge ${ }^{2}$, Atle Magnar Bones ${ }^{2}$, Zhenbiao Yang $^{1}$ \\ ${ }^{1}$ Department of Botany and Plant Sciences, Center for Plant Cell Biology, Institute of Integrative Genome Biology, University of \\ California, Riverside, California 92521, USA; ${ }^{2}$ Department of Biology, University of Science and Technology, N-7491 Trondheim, \\ Norway
}

Rho GTPases are molecular switches that act as key regulators of a many cellular processes, including cell movement, morphogenesis, host defense, cell division and gene expression. Rho GTPases are found in all eukaryotic kingdoms. Plants lack clear homologs to conventional Rho GTPases found in yeast and animals; instead, they have over time developed a unique subfamily, ROPs, also known as RAC. The origin of ROP-like proteins appears to precede the appearance of land plants. This review aims to discuss the evolution of ROP/RAC and to compare plant ROP and animal Rho GTPases, focusing on similarities and differences in regulation of the GTPases and their downstream effectors.

Cell Research (2006) 16:435-445. doi:10.1038/sj.cr.7310055; published online 15 May 2006

Keywords: Rho GTPase, RhoGAP, RhoGEF, RhoGDI, cytoskeleton, NADPH oxidase, RIC

\section{Introduction}

The Ras superfamily of small GTPases constitutes a large class of monomeric GTP-binding proteins with a molecular weight of $20-40 \mathrm{kDa}$ found in all eukaryotes [1]. The basic property of these proteins is their ability to bind and hydrolyze GTP. Thus, the GTPases can exist in two states: they are active when GTP is bound and inactive when GDP is bound. The ability to cycle between active and inactive states makes these proteins an ideal "molecular switch" for the transmission of discrete "on-off" signals in the cell [2].

\footnotetext{
Correspondence: Zhenbiao Yang ${ }^{1}$, Atle M Bones ${ }^{2}$

${ }^{1}$ Tel: 1-951-827-7351; Fax: 1-951-827-4437;

E-mail: yang@ucr.edu

${ }^{2}$ Tel: 1-47-7359-8692; Fax: 47-7359-6100;

E-mail: atle.bones@bio.ntnu.no

Abbreviations: CRIB (Cdc42/Rac-interactive binding); DH (Dbl homology); FH (formin homology); GAP (GTPase-activating protein); GDI (GDP dissociation inhibitor); GEF (GDP exchange factor); PH (Pleckstrin homology); PRONE (plant-specific Rop nucleotide exchanger); Rboh (Respiratory burst oxidase homolog); RIC (ROP-interactive CRIB-containing protein); RLK (Receptor-like kinase); ROP (Rho-related GTPase from plants); ROS (reactive oxygen species)
}

The Ras superfamily is classified into five families: Rab, Arf/Sar, Ran, Ras and Rho. Rab and Arf/Sar family GTPases function in intracellular vesicle trafficking; Ran is involved in nuclear transport, whereas the Ras and Rho family GTPases regulate signal transduction from the plasma membrane. Curiously, Ras GTPase homologs have not been found in viridiplantae (land plants and green algae). Research on Rho GTPases has mostly been focused on members of the Rac, Cdc42 and Rho subfamilies [3]. The Rho GTPase family in plants is unusual; no clear homologs to the Rac, Rho or Cdc42 subfamilies have been reported. Instead, plants contain a unique subfamily of Rho GTPases, called ROPs (Rho-related GTPase from plants) [4-7]. ROPs are sometimes referred to as RACs, because the primary amino acid sequences are most homologous to animal RACs. Being the only small GTPase family dedicated to signal transduction in plants, ROPs have been shown to be key regulators of a number of cellular processes. Some of the mechanisms by which ROPs receive or transmit signals are conserved between animals and plants, whereas others are plant specific. In this review, we examine the evolution of Rho GTPases in eukaryotic organisms and compare plant ROPs with Rho family GTPases from other organisms, looking at similarities and differences in regulation 
of GTPase activity and downstream pathways.

\section{Rho subfamilies}

In mammals, the Rho GTPase family consists of 22 members which can be divided into eight subgroups: Cdc42, Rac, Rho, Rnd, RhoD, RhoH, RhoBTB and Miro [8]. In plants, however, only two subgroups appear to exist: Miro and ROP/RAC. With a size of about $70 \mathrm{kDa}$, the Miro (mitochondrial rho) GTPases are not really small GTPases; these proteins contain two domains with similarity to small GTPases, as well as two $\mathrm{Ca}^{2+}$-binding EF hands [9]. The N-terminal GTPase domain shows significant similarity towards Rho GTPases. In yeast, the Miro homolog Gem1p is localized to the outer mitochondrial membrane [10]. Yeast cells lacking Gem1p contain globular, collapsed or grapelike mitochondria, indicating a role for Gem1p in regulating mitochondrial morphology. The Arabidopsis genome contains three genes encoding putative Miro homologs (At3g05310, At3g63150 and At5g27540). No results have yet been published on plant Miro homologs.

In contrast, the other subgroup of plant Rho-like GTPases, ROP/RAC, has been subject to intense research since their discovery more than ten years ago [11-13]. The ROP family in Arabidopsis comprises 11 members [7]. Plant ROP GTPases carry a number of conserved amino acid substitutions compared with animal Rho GTPases that identify them as a unique subgroup. These substitutions are found in the GTP binding motifs, as well as in several of the exposed loops. The difference is particularly evident in the insert region, an exposed loop corresponding to residues 124-135 in human Rac1, where ROPs contain a two- to four-amino acid deletion compared to animal Rho GTPases. Figure 1 summarizes mechanisms for regulation of ROP activity and downstream effectors.

\section{Proteins regulating Rho GTPase activity}

The intrinsic GTPase activity of Rho GTPases hydrolyzes GTP to GDP, leading to conformational changes that abolish interactions with downstream effectors. The switch back to the GTP-bound state occurs when GDP is released and replaced by GTP.

The cycling of Rho GTPases between GTP- and GDPbound states is regulated by three protein classes: GTPaseactivating proteins (GAPs), GDP dissociation inhibitors (GDIs) and GDP exchange factors (GEFs).

\section{RhoGAP}

GAPs increase the intrinsic GTPase activity by stabilizing a transition state in which a hydrolytic water molecule is positioned proximal to the terminal phosphate group of GTP [14]. Essential for the GAP activity is a conserved arginine residue (the "arginine finger") that is inserted

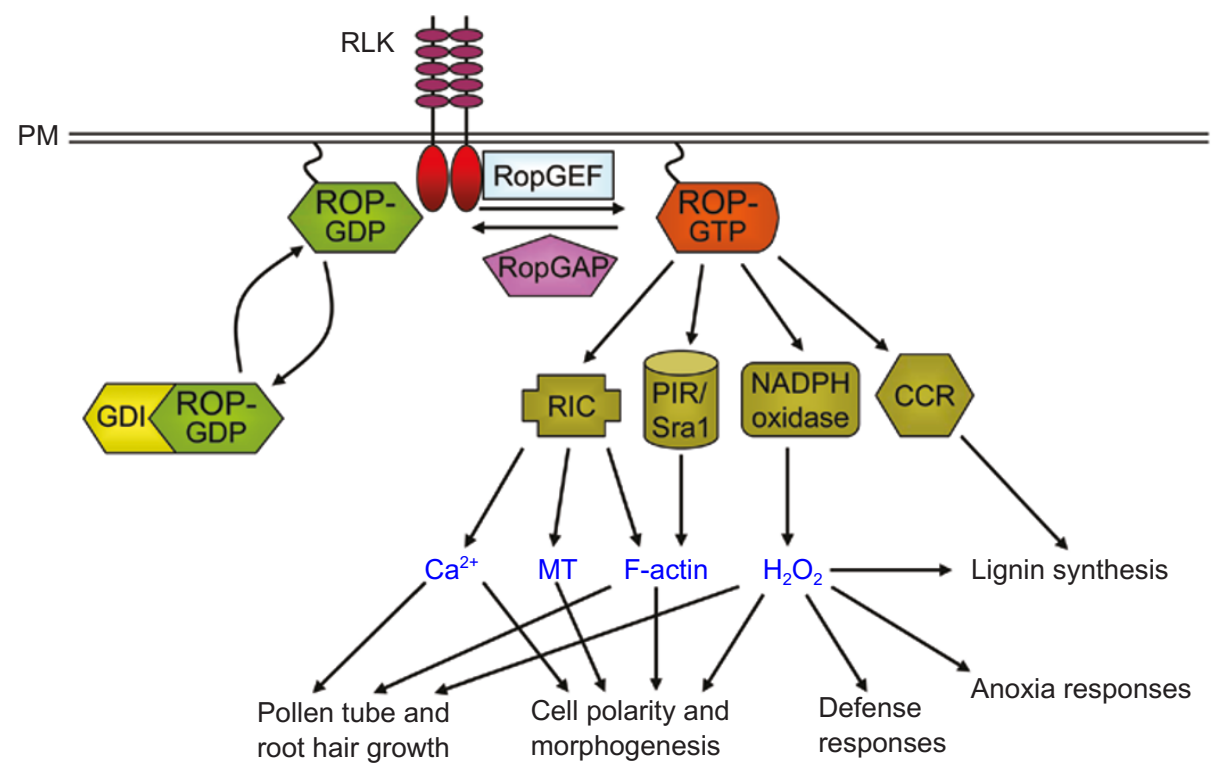

Figure 1 Overview of ROP signaling pathways. Extracellular signals activate a receptor-like kinase at the cell surface. RLK-mediated activation of RopGEF induces the exchange of ROP-bound GDP with GTP. GTP-bound, active ROP interact with a number of effectors to regulate a wide variety of cellular processes. ROP signaling is attenuated by RhoGAPs, whereas RhoGDIs may be important for both spatial and temporal regulation of ROP activity. 
into the GTPase active site. The best characterized among RhoGAP-like protein families in plants is the RopGAP family, consisting of six members in Arabidopsis $[15,16]$. RopGAPs have an unusual domain organization, with a Cdc42/Rac-interactive binding (CRIB) motif adjacent to the RhoGAP domain. In animals, the CRIB motif has been found exclusively in Rac and Cdc42 effectors. In vitro assays have shown that RopGAP1 enhances the GTPase activity of ROP1; the CRIB motif is necessary both for this activity and for effective binding to ROP [16]. The CRIB motif of the RopGAPs may stabilize the transition state, thereby facilitating the GAP activity. Another possibility is that the CRIB motif could mediate crosstalk between different ROPs; binding of one ROP to the RopGAP CRIB could activate the RopGAP, leading to inactivation of another ROP.

One RopGAP has been shown to take part in a ROP rheostat regulating the response to oxygen deprivation in $\mathrm{Arabi}$ dopsis [17]. Under anoxic conditions, an unidentified ROP induces production of hydrogen peroxide $\left[\mathrm{H}_{2} \mathrm{O}_{2}\right]$ through the activation of NAPDH oxidase (discussed below). $\mathrm{H}_{2} \mathrm{O}_{2}$ triggers the expression of alcohol dehydrogenase (ADH), an enzyme involved in ethanolic fermentation. A negative feedback loop to ROP is provided by the $\mathrm{H}_{2} \mathrm{O}_{2}$-induced expression of RopGAP4, which terminate ROP signaling, thereby preventing damaging production of reactive oxygen species (ROS).

In addition to the RopGAP family, the Arabidopsis genome encodes two uncharacterized protein families containing a conserved RhoGAP domain. A small family of putative RhoGAPs, consisting of three members in Arabidopsis, contains an N-terminal Pleckstrin homology $[\mathrm{PH}]$ domain, followed by a RhoGAP domain (Vernoud V, Hwang J-U, Yang Z, unpublished data). PH domains are 100-120 amino acid protein modules with the ability to bind phosphoinositides, and are found in a diverse set of proteins, amongst them RhoGEFs and RhoGAPs [18]. The domain organization indicates that lipid signaling may be important for regulation of the activity and/or localization of these RhoGAPs. The GAP activity has still not been shown biochemically for members of this family. A third putative RhoGAP family, represented by a single gene (At5g61530) in Arabidopsis, does not contain any known protein motifs besides the GAP domain.

\section{RhoGDI}

RhoGDIs regulate Rho GTPases a) by inhibiting the dissociation of bound nucleotide from their binding GTPase partners; b) by inhibiting GTP hydrolysis and interaction with GAPs, GEFs and effectors; and c) by extracting Rho GTPases from the plasma membrane, thereby maintaining a cytoplasmic pool of GTPases [19, 20]. The Arabidopsis genome encodes three RhoGDI homologs with high similarity to mammalian RhoGDI, except for the N-terminal 50 amino acids. Interaction between ROP and RhoGDI has been shown in Arabidopsis and tobacco [21, 22]. The residues Asp45 and Asp185 of human RhoGDI-1, which has been shown to be necessary for inhibition of GDP dissociation from Cdc42 [23] are conserved in all three Arabidopsis RhoGDIs. Recently, the Arabidopsis RhoGDI1 has been found to be involved in root hair development, probably through regulation of ROP2 localization [24]. The root hair cells of supercentipedel (scnl) plants, in which RhoGDI1 function is disrupted, develop multiple growth sites along the outer face. Wild type root hair cells produce only one growth site per cell. Studies of ROP2 localization showed that whereas ROP2 becomes restricted to the root hair growth site in wild type plants, it is ectopically localized at the cell surface of scn 1 root hair cells.

\section{RhoGEF}

RhoGEFs facilitate the exchange of GDP with GTP, thereby switching the GTPase to its active state. Two classes of RhoGEFs have been identified in animals. Members of the Dbl family of RhoGEFs all contain a Dbl homology [DH] domain followed by a $\mathrm{PH}$ domain [25]. Surprisingly, no DH domains have yet been reported in plants. The other RhoGEF family is characterized by two conserved domains called Dock homology region (DHR) 1 and 2 [25]. The first member of this family that was characterized as a RhoGEF was Dock180, also termed Dock1 [26]. Arabidopsis contains a single gene encoding a Dock-like protein called SPIKE1 (SPK1] [27]. spk1 mutant plants show defects in actin filament and microtubule organization and are seedling-lethal. However, GEF activity towards ROP has still not been shown for SPIKE1. Homologs of the Dock-associated protein ELMO are also present in plants.

Recently, a novel, plant-specific family of RhoGEFs termed RopGEF has been identified through yeast twohybrid screens using dominant negative Rop mutants as bait $[28,29]$. This family consists of 14 members in Arabidopsis that share a large central domain (315 amino acids), named PRONE (plant-specific Rop nucleotide exchanger) or DUF315, necessary for RopGEF activity. The plant specificity of the RopGEFs is supported by the inability of RopGEF1 to increase nucleotide exchange in human Rac1. In contrast, the DH-PH region of the human RhoGEF Tiam1 showed GEF activity towards Arabidopsis ROP4, although at a lower level compared with RopGEF1 [28]. Overexpression of RopGEF1 in pollen tubes resulted in depolarization of pollen tube growth, similar to the phenotype observed when a constitutively active (CA) ROP1 mutant is expressed [29]. Expression of a dominant 
negative (DN) ROP1 mutant suppressed the RopGEF1 overexpression phenotype, further supporting a role for ROPGEF1 as an upstream activator of ROP1.

\section{Signaling to RopGEFs - a role for receptor-like kinases}

How are extracellular signals passed through the plasma membrane to the Rho GTPases? In animal cells, transmembrane receptor tyrosine kinases (RTKs) constitute a large and diverse family of cell surface receptors that play important roles in fundamental cell processes [30]. A number of RhoGEFs (and, to a lesser extent, RhoGAPs) are activated by RTKs, leading to activation or inactivation of downstream Rho GTPases [31, 32]. However, receptor tyrosine kinases appear to be absent in plants. Instead, a large family of receptor-like kinases (RLKs) with diverse extracellular domains and a cytoplasmic serine-threonine kinase domain has been found in plants; members of this family are involved in a plethora of signaling processes, such as hormonal responses, pathogen recognition, meristem development and self-incompatibility $[33,34]$.

Interestingly, a RopGEF was isolated in a yeast twohybrid screen with the pollen-specific tomato RLKs LePRK1 and LePRK2 [35]. The RopGEF, termed KPP (kinase partner protein), interacted with the cytoplasmic domain of LePRK1 and LePRK2 and was phosphorylated in vivo, suggesting that KPP may be a substrate of LePRK1/ LePRK2. Overexpression of KPP in pollen led to depolarized growth of the pollen tube, resulting in balloon-shaped pollen tube tips.

ROP has also been implicated in signaling downstream of the RLK CLAVATA1 (CLV1). CLV1 is part of a protein complex regulating the balance between cell differentiation and cell division in aerial meristems; clv1 mutant plants show ectopic accumulation of stem cells at shoot and flower meristems [36]. An unidentified ROP GTPase was immunologically detected in the $450 \mathrm{kDa}$ active CLV1 complex, together with the protein phosphatase KAPP. Still, one or more components of the complex were not identified. By analogy to the PRK-KPP interaction, it is not unlikely that a RopGEF might link ROP to the CLV1 complex.

\section{Plant ROP vs. animal Rho effectors and downstream processes}

\section{The actin cytoskeleton}

In both animals and plants, Rho GTPases play pivotal roles in regulation of the actin cytoskeleton [38-40]. In mammalian cells, cell shape, polarity and movement are governed by the coordinated activity of different Rho GTPases. Expression of constitutively active and dominant negative mutants of $\mathrm{Cdc} 42$, Rac and Rho induce different structures when expressed in tissue culture [41-43].
Activation of Cdc42 leads to the generation of actin-rich finger-like protrusions called filopodia. Active Rac induce actin-rich surface protrusions called lamellipodia. Rho activation leads to the assembly of actin:myosin filaments known as stress fibers. During cell migration, Rac and Cdc42 localize to the leading edge of the cells. Rac-induced actin polymerization is believed to be important for membrane protrusion at the leading edge, whereas $\mathrm{Cdc} 42$ regulates polarity and direction of cell movement. Rho, acting at the rear of the cell, generates contractile actin: myosin filaments that regulate cell retraction at the tail end of the migrating cell.

Plant cells, being surrounded by rigid cell walls, do not move or grow through the protrusion of a leading cell edge. Instead, plant cell expansion is driven by turgor pressure and controlled by cell wall properties and the cytoskeleton $[44,45]$. A growing body of evidence also points to ROP as a key player in the generation of cell polarity in plant cell growth and morphogenesis. ROPs have been shown to regulate polar growth and cell morphogenesis in several cell systems including pollen tubes, developing root hairs, and leaf pavement cells [46-54].

Arp $2 / 3$ is a protein complex consisting of seven subunits; the Arp2 and Arp3 subunits share similarity with conventional actins. The Arp2/3 complex associates with the sides of existing actin filaments and initiates a new filament branching out at an angle of $70^{\circ}$ relative to the orientation of the parent filament. Rac and Cdc42 regulate Arp $2 / 3$ activity through the related WAVE and WASP families, respectively. WASP and WAVE proteins all contain a C-terminal domain, VCA, which initiates actin polymerization by binding actin monomers and the Arp $2 / 3$ complex, thereby bringing them together $[55,56]$. The diverging N-terminal regulatory domains of WASP and WAVE domains account for their different modes of regulation. WASP homologs have not been identified in plants, and will not be discussed further. In mammals, Rac and the adapter protein Nck regulate the three WAVE family members through a pentameric protein complex called the WAVE or SCAR complex [57]. In addition to WAVE, the complex includes PIR121/Sra-1, Nap1/Hem1, Abi1/Abi2 and HSPC300.

Homologs of all subunits of the Arp2/3 and WAVE complex have been identified in Arabidopsis [58, 59]. A plant Arp $2 / 3$ complex has yet to be shown biochemically to exhibit the same properties as its counterparts in other eukaryotic organisms. However, Arabidopsis homologs of the Arp3 and ArpC2 subunits were able to complement mutants for the corresponding genes in yeast $[60,61]$. Mutants of Arabidopsis ARP2, ARP3, APRC2a, ARPC5, NAP1 and PIR121/SRA1, as well as BRK1 RNAi plants, all show highly similar phenotypes, characterized by cell 
expansion defects in different epidermal cell types [46, 60-68]. Furthermore, T-DNA knockout mutants for one of four Arabidopsis WAVE/SCAR homologs show similar, but milder phenotypes $[69,70]$. The most striking phenotype is seen in trichomes, which are large, unicellular structures protruding from the leaf epidermis. Mutant trichomes display randomized cell expansion in these mutants, resulting in phenotypes that are mimicked by treatment with actin-depolymerizing drugs [71, 72]. Yeast two-hybrid experiments show that ROP2 interacts with PIR121/SRA1, suggesting that WAVE activity in plants may be regulated by ROP [46]. The fact that this interaction is conserved between animals and plants supports the hypothesis that ROP GTPases originated from a Rac-like ancestor. Overexpression of $C A$-rop 2 results in a trichome phenotype resembling the ARP2/3 and WAVE complex mutants [48], but the functional relationship between ROP and the WAVE-ARP2/3 pathway remains to be established.

The Arp $2 / 3$ and WAVE complex mutant phenotypes are surprisingly subtle compared with corresponding mutants in other organisms. Disruption of Arp2 and ARPC1 in budding yeast is lethal $[73,74]$. Mutation or RNAi suppression of Arp2/3 or WAVE complex subunits in Drosophila and $C$. elegans all result in embryolethality $[75,76]$. Actin structures produced by the ARP $2 / 3$ complex apparently plays a non-essential role in plants, only being important for proper morphogenesis of cells with complex growth patterns. Instead, formins may act as the major actin-nucleating protein class in plants and might be regulated by ROPs. It is also possible that plants have evolved unique ROP-mediated mechanisms for actin nucleation.

Formins are actin-nucleating proteins with the ability to stimulate de novo polymerization of actin filaments [77, 78]. They are conserved throughout eukaryota, and are recognized by the presence of a highly conserved formin homology 2 (FH2) domain, which is essential for actin filament nucleation. Most formins contain a proline-rich, profilin-binding FH1 domain adjacent to the FH2 domain. A subclass of formins, the Diaphanous-related formins (Drfs), contains an N-terminal Rho GTPase-binding domain. Binding of Rho GTPases releases autoinhibitory interactions, thereby activating Drf.

The formin family appears to be expanded in plants compared to animals; the Arabidopsis genome encodes 21 putative formin homologs [79], whereas only 9 genes encoding formins have been identified in mammalian genomes so far [80]. Most of the plant formins contain a FH1 domain in addition to the FH2 domain. However, no plant formins appear to have a GTPase-binding domain, suggesting that ROP GTPases are not directly involved in regulation of formin activity. Still, one cannot exclude the possibility that ROP participates in formin regulation through a plant-specific mechanism not yet discovered.

Apart from the conserved actin nucleation mechanisms, ROPs might regulate a novel mechanism for the assembly of the actin cytoskeleton $[47,50]$. Evidence indicates that ROP1 activates the assembly of a dynamic form of tip-localized actin microfilaments required for pollen tube growth [49]. However, knocking out the Arp $2 / 3$ complex has no effects on pollen tube growth, suggesting an Arp2/3 complex independent mechanism is involved in the assembly of the tip actin (Li and Yang, unpublished data). Similarly, ROP2dependent cortical diffuse F-actin remains in the pavement cells of Arabidopsis leaves defective in the Arp2/3 complex [66]. As discussed below, in both pollen tubes and pavement cells, the assembly of cortical F-actin is activated by a plant-specific ROP effector protein, RIC4.

\section{RICs (ROP-interactive CRIB-containing proteins)}

The CRIB motif (also known as GBD, or GTPase-binding domain) is used for GTP-dependent interaction with Rac and Cdc 42 subfamily members by a number of Rho effector proteins in animal cells [81]. The Pak (p21-activated kinase) kinases are a family of CRIB motif-containing serine-threonine kinases that phosphorylate a wide variety of substrate proteins upon binding of activated Rac or Cdc42 $[82,83]$. Pak kinases participate in regulation of gene expression through MAP kinase pathways, and organization of both actin and microtubule cytoskeleton. Most eukaryotes encode one or more Pak homologs, but none are found in plants. In fact, only two protein families have retained a CRIB motif in plants, none of which are conserved in animals. One is the RopGAP family discussed above. The other is a family of small, plant specific proteins called RICs (ROP-interactive CRIB-containing proteins) [84]. In Arabidopsis, the RIC family comprises 11 members, which based on sequence similarity can be sorted into five subfamilies based on sequence similarity [84]. RIC's function has been studied in two model systems: pollen tubes [50] and leaf pavement cells [47]. ROP1 regulates pollen tube growth through coordination of two counteracting pathways: activation of RIC4 promotes assembly of apical F-actin in the pollen tube tip, whereas activation of RIC3 leads to disassembly of the apical F-actin through a $\mathrm{Ca}^{2+}$ dependent process [50]. In turn, these two pathways appear to provide positive (RIC4) or negative (RIC3) feedback signaling to ROP1, leading to an oscillation of ROP1 activity at the pollen tube tip that is correlated with tip growth oscillation [85]. The generation of the jigsaw puzzle-like shape of leaf pavement cells appears to be at least partly regulated by ROP-RIC signaling pathways [47]. RIC4 acts downstream of ROP2 and ROP4 to promote the assembly of cortical actin filaments in developing lobes, thus facilitating localized growth of these lobes. In contrast, RIC1 is 
inactivated by ROP2/4 binding. RIC1 activity seems to be restricted to the neck regions, where it promotes formation of well-ordered cortical microtubules that promotes narrow neck morphology. Furthermore, RIC1-mediated microtubule arrays suppress the ROP-RIC4-actin pathway. Thus, the concerted action of these two counteracting pathways contributes to the generation of interdigitating lobes and indentations of pavement cells.

\section{Regulation of NADPH oxidase}

Production of ROS is an important part of host defense in animals. During phagocytosis of invading microbes, phagocytes produce superoxide anion $\left[\mathrm{O}_{2}^{-}\right]$, which is subsequently converted to other ROS; these compounds contribute to the killing of the phagocytosed microbe [86]. The enzymatic activity for production of $\mathrm{O}_{2}^{-}$is provided by the protein complex NADPH oxidase. The catalytic component is the membrane protein gp91 ${ }^{\text {phox }}$, also termed Nox [87]. Other subunits include another membrane protein, $\mathrm{p} 22^{\text {phox }}$, and the cytosolic components $\mathrm{p} 40^{\text {phox }}, \mathrm{p} 47^{\text {phox }}$ and $\mathrm{p} 67^{\text {phox }}$ Finally, active Rac2 is necessary for NADPH oxidase activity in phagocytes, interacting with both p67 $7^{\text {hox }}[88]$ and gp9 $1^{\text {phox }}[89]$. The NADPH oxidase is expressed in many cell types, and superoxide production has been implicated in a number of cellular processes besides host defense.

Homologs of gp91 $91^{\text {phox }}$ have been identified in several plant species [90-92]. The Arabidopsis genome encodes ten putative gp9 $1^{\text {phox }}$ homologs, termed Respiratory burst oxidase homolog (Rboh) [93]. With the exception of ROP GTPases, none of the other components of the NADPH complex appears to be conserved in plants. Thus, regulation of NADPH oxidase activity is different in plants and animals. Plant Rbohs all contain a cytosolic N-terminal region with two $\mathrm{Ca}^{2+}$-binding $\mathrm{EF}$ hands [91]; this region is absent in the phagocytic gp91 ${ }^{\text {phox }}$, but is found in other members of the mammalian Nox family [94]. In vitro assays indicate that plant Rbohs are able to produce superoxide in the absence of cytosolic factors and are stimulated directly

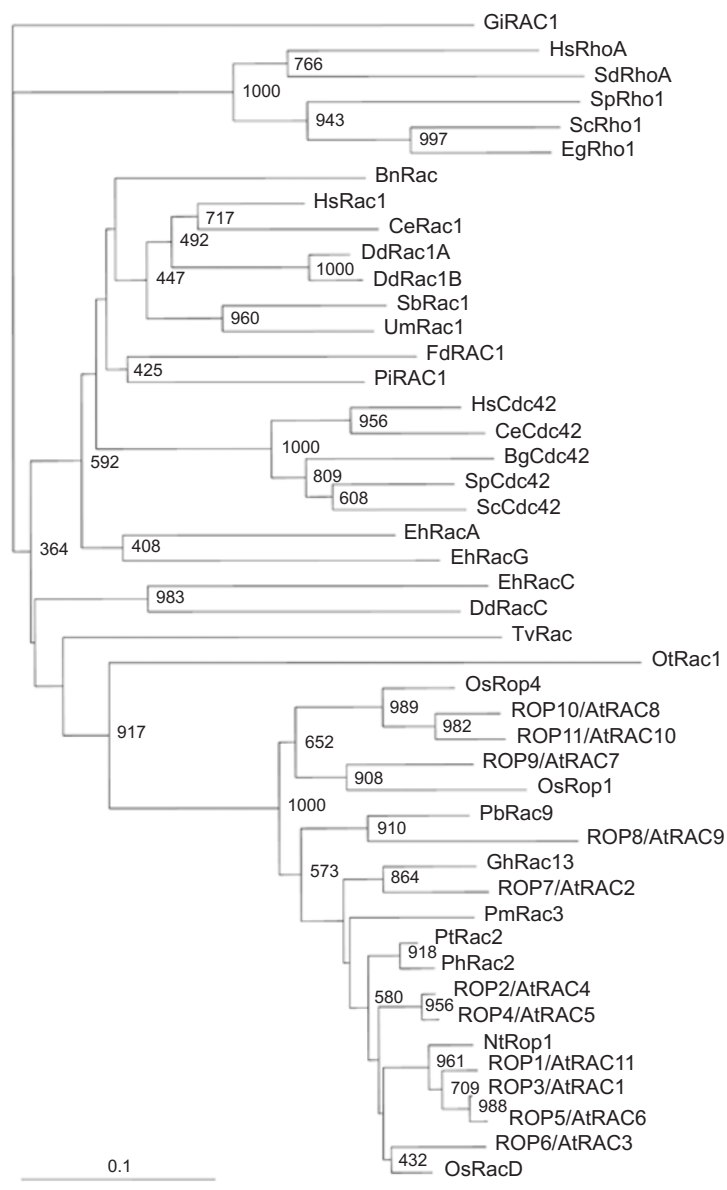

Figure 2 A phylogram of Rho GTPases in different eukaryotic organisms. The protein alignment of the Rho-family proteins was produced with the GeneDoc program version 2.4.017 and the multiple sequence file was imported into the Clustal X program. The hypervariable $\mathrm{N}$ - and $\mathrm{C}$-terminal ends were excluded. Protein weight matrixes of the PAM series were used to calculate the distances and an unrooted neighbor-joining $(\mathrm{N}-\mathrm{J})$ tree was created using the neighbor-joining algorithm (Saitou 105). Bootstrapping of the N-J tree was done with 1000 bootstrap trials. The tree was rooted with the Rac gene from the amitochondriate protist Giardia lamblia and the tree was visualized using the Treeview program [106].

The figure shows the relationship between some of the characteristic protein families within the larger Rho-family. The Rac protein from the prasinophyte Ostreococcus tauri is an out-group for the plant RAC/ROP proteins and reinforce the close phylogenetic relationship between prasinophytes and the streptophyta. The Cdc42 and RhoA/Rhol proteins are exclusively found within the metazoa/fungi clade and have high bootstrap support. The Rac proteins show a broader phylogenetic distribution and do also include members from the protists; stramenopiles, mycetozoa and cercozoa, suggesting that this group has the deepest root among the extended Rho-family proteins.

Abbreviations for prefixes: At: Arabidopsis thaliana, Bg: Blumeria graminis, Bn: Bigelowiella natans (acc: DR041190), Ce: Caenorhabditis elegans, Dd: Dictyostelium discoideum, Eg: Eremothecium gossypii, Eh: Entamoeba histolytica, Fd: Fucus distichus, Gi: Giardia lamblia, Hs: Homo sapiens, Nt: Nicotiana tabacum, Os: Oryza sativa, Ot: Ostreococcus tauri (acc: CR954206.2), Pb: Populus balsamifera (acc: CN550455), Ph: Physcomitrella patens, Pi: Phytophthora infestans (acc: CV947213), Pm: Picea mariana, Pt: Pinus taeda, Sb: Suillus bovines, Sc: Saccharomyces cerevisiae, Sd: Suberites domuncula, Sp: Schizosaccharomyces pombe, Tv: Trichomonas vaginalis (acc: AAP79439), Um: Ustilago maydis. 
Table 1 Distribution of Rho GTPases and Rho regulatory protein domains in eukaryotic lineages

\begin{tabular}{|c|c|c|c|c|c|c|}
\hline & Rho & RhoGAP & RhoGDI & $\begin{array}{l}\text { RhoGEF } \\
\text { DH }\end{array}$ & $\begin{array}{l}\text { RhoGEF } \\
\text { Dock/CZH }\end{array}$ & $\begin{array}{l}\text { RhoGEF } \\
\text { PRONE }\end{array}$ \\
\hline Plants (Arabidopsis thaliana) & $\mathrm{X}$ & $\mathrm{X}$ & $\mathrm{X}$ & & $\mathrm{X}$ & $\mathrm{X}$ \\
\hline Fungi (Aspergillus fumigatus) & $\mathrm{X}$ & $\mathrm{X}$ & $\mathrm{X}$ & $\mathrm{X}$ & $\mathrm{X}$ & \\
\hline Metazoa (Homo sapiens) & $\mathrm{X}$ & $\mathrm{X}$ & $\mathrm{X}$ & $\mathrm{X}$ & $\mathrm{X}$ & \\
\hline Alveolata (Paramecium tetraurelia) & $X$ & $X$ & $X$ & & & \\
\hline Cercozoa (Bigelowiella natans) & $\mathrm{X}$ & & $\mathrm{X}$ & $\mathrm{X}$ & & \\
\hline Diplomonadida (Giardia lamblia) & $\mathrm{X}$ & $\mathrm{X}$ & $X$ & & & \\
\hline Entamoebidae (Entamoeba histolytica) & $\mathrm{X}$ & $\mathrm{X}$ & $\mathrm{X}$ & $\mathrm{X}$ & $\mathrm{X}$ & \\
\hline Rhodophyta (Porphyra haitanensis) & & $\mathrm{X}$ & & & & \\
\hline
\end{tabular}

by $\mathrm{Ca}^{2+}[95]$. However, ROP also appears to be involved in regulation of Rboh activity $[17,96]$. In rice, a pathway leading from heterotrimeric $\mathrm{G}$ protein via OsRac1 to NADPH oxidase confers disease resistance towards pathogens such as rice blast fungus [96-98]. Although the precise mechanism of this pathway has not yet been resolved, Kawasaki et al. [99] mention as unpublished results that ROP regulates Rboh activity through direct interaction. It will be interesting to see whether this interaction is similar to the interaction between Rac and gp9 $91^{\text {phox }}$ in mammalian cells.

\section{Novel ROP interactions in plants}

Besides RIC, only a few ROP-effector interaction pairs are not conserved in other phyla. Recently, rice cinnamoyl-CoA reductase1 (OsCCR1), an enzyme involved in lignin biosynthesis, was shown to interact with OsRac1 in a GTP-dependent manner [99]. Interaction with OsRac1 induced the enzymatic activity of OsCCR1 in vitro. $\mathrm{H}_{2} \mathrm{O}_{2}$, a secondary product of NADPH oxidase, is required for polymerization of monolignols. OsRac1 may therefore control lignin synthesis through regulation of both OsCCR1 and NADPH oxidase.

Arabidopsis UDP-glucose transferase 1 (UGT1) is part of a protein complex including callose synthase [100]. UGT1 interacts with GTP-bound, but not GDP-bound ROP1, suggesting that UGT1 may be a ROP effector. Interestingly, yeast Rholp regulates callose synthase activity through direct interaction with the catalytic subunit [101].

\section{An evolutionary view of Rho GTPases and their functional}

partners

The Rac/Rho protein is of ancient origin and can be found in most eukaryotic lineages (Table 1). It evolved at a time when eukaryotic organisms were unicellular, and their later diversification into distinct gene families, notably the Rho and Cdc42 families, happened after the establishment of major eukaryotic divisions. Thus, the Rac/Rho proteins have developed independently within the individual eukaryotic lineages for hundreds of millions of years. Many eukaryotic species have evolved large Rac/Rho gene families; humans for instance have $22 \mathrm{Rac} / \mathrm{Rho}$ genes [8], and Dictyostelium discoideum and Entamoeba histolytica have 18 and 26, respectively [102]. The amoebozoa diverged from the animal-fungal lineage after the plant kingdom had developed, and the phylogenetic analyses of the Raclike genes in these organisms (Figure 2) clearly show this relationship. Phylogenetic analyses of the amoebae also suggest a similar close relationship between Dictyostelium and Entamoeba [103]. The high diversity and distinct gene families within the Rac/Rho genes in Dictyostelium discoideum and Entamoeba histolytica suggest that these genes evolved at an early stage during evolution. Only one of the Rac/Rho subfamilies within these organisms, the RacC homologues, suggests a monophyletic origin; the others are unique for each organism. The appearance of $\mathrm{Cdc} 42$ and Rho proteins in fungi and metazoa happened at a later stage in evolution, long after the animal-fungal lineage split from the viridiplantae. Due to the early split of viridiplantae from the animal-fungal-amoebozoa lineage, Rho-family genes in plants have developed novel features 
and modes of regulation. This may also explain why plants lack small GTPases and GTPase regulatory proteins found in animals and fungi. Rho-like genes do not exist in the green algae Chlamydomonas (Winge, unpublished data). However, in the prasinophyte Ostreococcus tauri, which is thought to be more closely related to plants (streptophyta), a gene encoding a GTPase related to plant RAC/ROP has recently been sequenced (Figure 2). In plants, the ROP/RAC proteins have just recently begun to split up into distinct gene families. The first terrestrial plants probably just had one single $R O P / R A C$ gene. One of the first major divisions happened after the appearance of the Spermatophyta. This division was caused by a mutation in a $R O P / R A C$ gene resulting in an extra $\mathrm{C}$-terminal exon which destroyed the native prenylation site (ROP/RAC group II) [7,104]. Later gene duplications have resulted in several distinct sub-groups of $R O P / R A C$ genes in monocotyledons and dicotyledons.

The effectors of Rho/Rac proteins, RhoGAP, RhoGDI and RhoGEF (Dbl homology type), have co-evolved with the Rac/Rho proteins over hundreds of millions of years. The DH domain RhoGEF is found in several eukaryotic lineages (Metazoa, Fungi, Mycetozoa, Entamoebidae, Cercozoa and Stramenopiles), but is missing in Streptophyta and has apparently also been lost (or did not develop) in Euglenozoa, Parabasalidea, Diplomonadida, Rhodophyta and Alveolata (Table 1). In contrast, a distinct family of RhoGEFs, RopGEFs, has co-evolved with plant ROPs/ RACs [28, 29]. When comparing the evolution of the Rac/Rho regulatory proteins, there are also other distinct differences. While the RhoGDIs always exist as a single domain protein, the RhoGAPs and RhoGEFs are often integral parts of multi-domain proteins. More than 20 different domains and motifs have been found associated with RhoGAP proteins, and close to 30 domains and motifs together with RhoGEFs [14, 25]. Some of these motifs, such as PH, ArfGAP, RasGEF, DEP, BAR and myosin head, are present in both RhoGAP and RhoGEF proteins. The many and diverse RhoGAP and RhoGEF proteins in Dictyostelium and Entamoeba imply that their Rac genes evolved and diversified much earlier than in plants. Similarly, the complexity of RhoGAP and RhoGEF proteins in metazoa and fungi reflects the early diversification of Rac/Rho genes in these organisms and shows that the $\mathrm{Rac} / \mathrm{Rho} / \mathrm{Cdc} 42$ proteins began to acquire specific functions before the division between metazoa and fungi. The diversification of $R O P / R A C$ genes in Streptophyta has appeared late in evolution and may explain why ROP/RAC GAPs still have a relative simple domain organization compared to the multi-domain RhoGAPs found in metazoa, fungi and amoebas. As a comparison, plants have just two described domains associated with their GAP proteins, CRIB [84] and
PH (Vernoud V, Hwang J-U, Yang Z, unpublished data). However, plants most likely have evolved novel RAC/ROP regulators and pathways yet to be discovered.

\section{Summary and perspectives}

Although phylogenetic analyses suggest that ROP/RAC proteins form a distinct Rho subfamily, several of the components involved in regulation of ROP activity are conserved between plants and other eukaryotes (RhoGAP, RhoGDI, Dock-type RhoGEF). However, a putative plant specific pathway for signaling from the plasma membrane to ROP, including a receptor-like kinase and a family of RhoGEFs unique to plants, has been identified. At least one mammalian Rac effector (PIR121/Sra1, a subunit of the WAVE complex) seems to be conserved in plants and to interact with ROP. Rho GTPases in animals and plants play similar roles in certain cellular processes, such as cell polarity and morphogenesis and production of ROS, although the precise mechanisms are not always conserved. Plant ROPs also regulate plant specific processes, such as lignin synthesis.

Still, many questions remain unanswered. What kind of extracellular signals activate RopGEFs through PRK-type RLKs? Are there other RLK subfamilies with activity towards RopGEFs? And how is the Dock-type RhoGEF SPK1 regulated? Although a functional role has been assigned to a few of the ROP regulatory proteins, knowledge on most members of these families is still very limited. Among the ROP effectors, the RICs are an enigmatic group of proteins. RICs have been shown to regulate F-actin, microtubules and $\mathrm{Ca}^{2+}$ levels; however, very little is known about the mechanisms by which RICs perform these functions and the crosstalk between the different pathways. Also, there may still be unidentified ROP effectors with exciting roles in processes such as meristem regulation, hormone responses and plant defense and cell morphogenesis.

\section{Acknowledgments}

This work was supported by the Biotechnology and Functional genomics (FUGE) programs of the Norwegian Research Council through grants NFR 159959, 164583 and 151991 (T B, P W and A M B), and by grants from the National Science Foundation, the Department of Energy and the US Department of Agriculture (Z Y).

\section{References}

1 Takai Y, Sasaki T, Matozaki T. Small GTP-binding proteins. Physiol Rev 2001; 81:153-208.

2 Bourne HR, Sanders DA, McCormick F. The GTPase superfamily: a conserved switch for diverse cell functions. Nature 1990; 
348:125-132.

3 Jaffe AB, Hall A. Rho GTPases: biochemistry and biology. Annu Rev Cell Dev Biol 2005; 21:247-269.

4 Li H, Wu G, Ware D, et al. Arabidopsis Rho-related GTPases: differential gene expression in pollen and polar localization in fission yeast. Plant Physiol 1998; 118:407-417.

5 Vernoud V, Horton AC, Yang Z, et al. Analysis of the small GTPase gene superfamily of Arabidopsis. Plant Physiol 2003; 131:1191-1208.

6 Winge P, Brembu T, Bones AM. Cloning and characterization of rac-like cDNAs from Arabidopsis thaliana. Plant Mol Biol 1997; 35:483-495.

7 Winge P, Brembu T, Kristensen R, et al. Genetic structure and evolution of RAC-GTPases in Arabidopsis thaliana. Genetics 2000; 156:1959-1971.

8 Sorokina EM, Chernoff J. Rho-GTPases: new members, new pathways. J Cell Biochem 2005; 94:225-231.

9 Fransson A, Ruusala A, Aspenstrom P. Atypical Rho GTPases have roles in mitochondrial homeostasis and apoptosis. J Biol Chem 2003; 278:6495-6502.

10 Frederick RL, McCaffery JM, Cunningham KW, et al. Yeast Miro GTPase, Gem1p, regulates mitochondrial morphology via a novel pathway. J Cell Biol 2004; 167:87-98.

11 Gu Y, Wang Z, Yang Z. ROP/RAC GTPase: an old new master regulator for plant signaling. Curr Opin Plant Biol 2004; 7:527536.

12 Yang Z. Small GTPases: versatile signaling switches in plants. Plant Cell 2002; 14 Suppl:S375-388.

13 Zheng ZL, Yang Z. The Rop GTPase: an emerging signaling switch in plants. Plant Mol Biol 2000; 44:1-9.

14 Moon SY, Zheng Y. Rho GTPase-activating proteins in cell regulation. Trends Cell Biol 2003; 13:13-22.

15 Borg S, Podenphant L, Jensen TJ, et al. Plant cell growth and differentiation may involve GAP regulation of Rac activity. FEBS Lett 1999; 453:341-345.

$16 \mathrm{Wu}$ G, Li H, Yang Z. Arabidopsis RopGAPs are a novel family of rho GTPase-activating proteins that require the $\mathrm{Cdc} 42 / \mathrm{Rac}-$ interactive binding motif for rop-specific GTPase stimulation. Plant Physiol 2000; 124:1625-1636.

17 Baxter-Burrell A, Yang Z, Springer PS, et al. RopGAP4-dependent Rop GTPase rheostat control of Arabidopsis oxygen deprivation tolerance. Science 2002; 296:2026-2028.

18 Lemmon MA, Ferguson KM, Abrams CS. Pleckstrin homology domains and the cytoskeleton. FEBS Lett 2002; 513:71-76.

19 DerMardirossian C, Bokoch GM. GDIs: central regulatory molecules in Rho GTPase activation. Trends Cell Biol 2005; 15:356-363.

20 Dovas A, Couchman JR. RhoGDI: multiple functions in the regulation of Rho family GTPase activities. Biochem J 2005; 390:1-9.

21 Bischoff F, Vahlkamp L, Molendijk A, et al. Localization of AtROP4 and AtROP6 and interaction with the guanine nucleotide dissociation inhibitor AtRhoGDI1 from Arabidopsis. Plant Mol Biol 2000; 42:515-530.

22 Kieffer F, Elmayan T, Rubier S, et al. Cloning of Rac and RhoGDI from tobacco using an heterologous two-hybrid screen. Biochimie 2000; 82:1099-1105.

23 Dransart E, Morin A, Cherfils J, et al. Uncoupling of inhibitory and shuttling functions of rho GDP dissociation inhibitors. J
Biol Chem 2005; 280:4674-4683.

24 Carol RJ, Takeda S, Linstead P, et al. A RhoGDP dissociation inhibitor spatially regulates growth in root hair cells. Nature 2005; 438:1013-1016.

25 Rossman KL, Der CJ, Sondek J. GEF means go: turning on RHO GTPases with guanine nucleotide-exchange factors. Nat Rev Mol Cell Biol 2005; 6:167-180.

26 Brugnera E, Haney L, Grimsley C, et al. Unconventional RacGEF activity is mediated through the Dock180-ELMO complex. Nat Cell Biol 2002; 4:574-582.

27 Qiu JL, Jilk R, Marks MD, et al. The Arabidopsis SPIKE1 gene is required for normal cell shape control and tissue development. Plant Cell 2002; 14:101-118.

28 Berken A, Thomas C, Wittinghofer A. A new family of RhoGEFs activates the Rop molecular switch in plants. Nature 2005; 436:1176-1180.

$29 \mathrm{Gu}$ Y, Li S, Lord EM, et al. Members of a Novel Class of Arabidopsis Rho Guanine Nucleotide Exchange Factors Control Rho GTPase-Dependent Polar Growth. Plant Cell 2006; 18:366-381.

30 Schlessinger J. Cell signaling by receptor tyrosine kinases. Cell 2000; 103:211-225.

31 Noren NK, Pasquale EB. Eph receptor-ephrin bidirectional signals that target Ras and Rho proteins. Cell Signal 2004; 16:655-666.

32 Van Aelst L, Cline HT. Rho GTPases and activity-dependent dendrite development. Curr Opin Neurobiol 2004; 14:297304.

33 Morris ER, Walker JC. Receptor-like protein kinases: the keys to response. Curr Opin Plant Biol 2003; 6:339-342.

34 Shiu SH, Bleecker AB. Receptor-like kinases from Arabidopsis form a monophyletic gene family related to animal receptor kinases. Proc Natl Acad Sci U S A 2001; 98:10763-10768.

35 Kaothien P, Ok SH, Shuai B, et al. Kinase partner protein interacts with the LePRK1 and LePRK2 receptor kinases and plays a role in polarized pollen tube growth. Plant J 2005; 42:492-503.

36 Clark SE, Williams RW, Meyerowitz EM. The CLAVATA1 gene encodes a putative receptor kinase that controls shoot and floral meristem size in Arabidopsis. Cell 1997; 89:575-585.

37 Trotochaud AE, Hao T, Wu G, et al. The CLAVATA1 receptorlike kinase requires CLAVATA3 for its assembly into a signaling complex that includes KAPP and a Rho-related protein. Plant Cell 1999; 11:393-406.

38 Hall A. Rho GTPases and the actin cytoskeleton. Science 1998; 279:509-514.

39 Hussey PJ, Ketelaar T, Deeks MJ. Control of the actin cytoskeleton in plant cell growth. Annu Rev Plant Biol 2006; Jan. Epub ahead of print (doi:10.1146/annurev.arplant.57.032905.105206)

40 Raftopoulou M, Hall A. Cell migration: Rho GTPases lead the way. Dev Biol 2004; 265:23-32.

41 Nobes CD, Hall A. Rho, rac, and cdc42 GTPases regulate the assembly of multimolecular focal complexes associated with actin stress fibers, lamellipodia, and filopodia. Cell 1995; 81:5362.

42 Ridley AJ, Hall A. The small GTP-binding protein rho regulates the assembly of focal adhesions and actin stress fibers in response to growth factors. Cell 1992; 70:389-399. 
43 Ridley AJ, Paterson HF, Johnston CL et al. The small GTPbinding protein rac regulates growth factor-induced membrane ruffling. Cell 1992; 70:401-410.

44 Bannigan A, Baskin TI. Directional cell expansion-turning toward actin. Curr Opin Plant Biol 2005; 8:619-624.

45 Smith LG, Oppenheimer DG. Spatial control of cell expansion by the plant cytoskeleton. Annu Rev Cell Dev Biol 2005; 21:271-295.

46 Basu D, El-Din El-Assal D, Le J, et al. Interchangeable functions of Arabidopsis PIROGI and the human WAVE complex subunit SRA1 during leaf epidermal development. Development 2004; 131:4345-4355.

$47 \mathrm{Fu} \mathrm{Y,} \mathrm{Gu} \mathrm{Y,} \mathrm{Zheng} \mathrm{Z,} \mathrm{et} \mathrm{al.} \mathrm{Arabidopsis} \mathrm{interdigitating} \mathrm{cell}$ growth requires two antagonistic pathways with opposing action on cell morphogenesis. Cell 2005; 120:687-700.

48 Fu Y, Li H, Yang Z. The ROP2 GTPase controls the formation of cortical fine F-actin and the early phase of directional cell expansion during Arabidopsis organogenesis. Plant Cell 2002; 14:777-794.

49 Fu Y, Wu G, Yang Z. Rop GTPase-dependent dynamics of tiplocalized F-actin controls tip growth in pollen tubes. J Cell Biol 2001; 152:1019-1032.

$50 \mathrm{Gu}$ Y, Fu Y, Dowd P, et al. A Rho family GTPase controls actin dynamics and tip growth via two counteracting downstream pathways in pollen tubes. J Cell Biol 2005; 169:127-138.

51 Jones MA, Shen JJ, Fu Y, et al. The Arabidopsis Rop2 GTPase is a positive regulator of both root hair initiation and tip growth. Plant Cell 2002; 14:763-776.

52 Kost B, Lemichez E, Spielhofer P, et al. Rac homologues and compartmentalized phosphatidylinositol 4, 5-bisphosphate act in a common pathway to regulate polar pollen tube growth. J Cell Biol 1999; 145:317-330.

$53 \mathrm{Li} \mathrm{H}$, Lin Y, Heath RM, et al. Control of pollen tube tip growth by a Rop GTPase-dependent pathway that leads to tip-localized calcium influx. Plant Cell 1999; 11:1731-1742.

54 Molendijk AJ, Bischoff F, Rajendrakumar CS, et al. Arabidopsis thaliana Rop GTPases are localized to tips of root hairs and control polar growth. Embo J 2001; 20:2779-2788.

55 Disanza A, Steffen A, Hertzog M, et al. Actin polymerization machinery: the finish line of signaling networks, the starting point of cellular movement. Cell Mol Life Sci 2005; 62:955970.

56 Pollard TD, Borisy GG. Cellular motility driven by assembly and disassembly of actin filaments. Cell 2003; 112:453-465.

57 Eden S, Rohatgi R, Podtelejnikov AV, et al. Mechanism of regulation of WAVE1-induced actin nucleation by Rac1 and Nck. Nature 2002; 418:790-793.

58 Brembu T, Winge P, Bones AM. Catching the WAVEs of plant actin regulation. Journal of Plant Growth Regulation 2005; 24:55-66.

59 Deeks MJ, Hussey PJ. Arp2/3 and SCAR: plants move to the fore. Nat Rev Mol Cell Biol 2005; 6:954-964.

60 El-Din El-Assal S, Le J, Basu D, et al. DISTORTED2 encodes an ARPC2 subunit of the putative Arabidopsis ARP2/3 complex. Plant J 2004; 38:526-538.

61 Le J, El-Din El-Assal D, Basu D, et al. Requirements for Arabidopsis ATARP2 and ATARP3 during epidermal development. Curr Biol 2003; 13:1341-1347.

62 Brembu T, Winge P, Seem M, et al. NAPP and PIRP encode subunits of a putative wave regulatory protein complex involved in plant cell morphogenesis. Plant Cell 2004; 16:2335-2349.

63 Deeks MJ, Kaloriti D, Davies B, et al. Arabidopsis NAP1 is essential for Arp2/3-dependent trichome morphogenesis. Curr Biol 2004; 14:1410-1414.

64 Djakovic S, Dyachok J, Burke M, et al. BRICK1/HSPC300 functions with SCAR and the ARP2/3 complex to regulate epidermal cell shape in Arabidopsis. Development 2006; 133:1091-1100.

65 El-Din El-Assal D, Le J, Basu D, et al. Arabidopsis GNARLED encodes a NAP125 homolog that positively regulates ARP2/3. Curr Biol 2004; 14:1405-1409.

66 Li S, Blanchoin L, Yang Z, et al. The putative Arabidopsis arp2/3 complex controls leaf cell morphogenesis. Plant Physiol 2003; 132:2034-2044.

67 Mathur J, Mathur N, Kernebeck B, et al. Mutations in actinrelated proteins 2 and 3 affect cell shape development in Arabidopsis. Plant Cell 2003; 15:1632-1645.

68 Mathur J, Mathur N, Kirik V, et al. Arabidopsis CROOKED encodes for the smallest subunit of the ARP2/3 complex and controls cell shape by region specific fine F-actin formation. Development 2003; 130:3137-3146.

69 Basu D, Le J, El-Essal Sel D, et al. DISTORTED3/SCAR2 is a putative arabidopsis WAVE complex subunit that activates the Arp2/3 complex and is required for epidermal morphogenesis. Plant Cell 2005; 17:502-524.

70 Zhang X, Dyachok J, Krishnakumar S, et al. IRREGULAR TRICHOME BRANCH1 in Arabidopsis encodes a plant homolog of the actin-related protein $2 / 3$ complex activator Scar/WAVE that regulates actin and microtubule organization. Plant Cell 2005; 17:2314-2326.

71 Mathur J, Spielhofer P, Kost B, et al. The actin cytoskeleton is required to elaborate and maintain spatial patterning during trichome cell morphogenesis in Arabidopsis thaliana. Development 1999; 126:5559-5568.

72 Szymanski DB, Marks MD, Wick SM. Organized F-actin is essential for normal trichome morphogenesis in Arabidopsis. Plant Cell 1999; 11:2331-2347.

73 Morrell JL, Morphew M, Gould KL. A mutant of Arp2p causes partial disassembly of the Arp2/3 complex and loss of cortical actin function in fission yeast. Mol Biol Cell 1999; 10:42014215.

74 Winter DC, Choe EY, Li R. Genetic dissection of the budding yeast Arp2/3 complex: a comparison of the in vivo and structural roles of individual subunits. Proc Natl Acad Sci U S A 1999; 96:7288-7293.

75 Hudson AM, Cooley L. A subset of dynamic actin rearrangements in Drosophila requires the Arp2/3 complex. J Cell Biol 2002; 156:677-687.

76 Sawa M, Suetsugu S, Sugimoto A, et al. Essential role of the C. elegans Arp2/3 complex in cell migration during ventral enclosure. J Cell Sci 2003; 116:1505-1518.

77 Wallar BJ, Alberts AS. The formins: active scaffolds that remodel the cytoskeleton. Trends Cell Biol 2003; 13:435-446.

78 Zigmond SH. Formin-induced nucleation of actin filaments. Curr Opin Cell Biol 2004; 16:99-105.

79 Deeks MJ, Hussey PJ, Davies B. Formins: intermediates in signal-transduction cascades that affect cytoskeletal reorganization. Trends Plant Sci 2002; 7:492-498. 
80 Li F, Higgs HN. The mouse Formin mDial is a potent actin nucleation factor regulated by autoinhibition. Curr Biol 2003; 13:1335-1340.

81 Hoffman GR, Cerione RA. Flipping the switch: the structural basis for signaling through the CRIB motif. Cell 2000; 102:403406.

82 Bokoch GM. Biology of the p21-activated kinases. Annu Rev Biochem 2003; 72:743-781.

83 Hofmann C, Shepelev M, Chernoff J. The genetics of Pak. J Cell Sci 2004; 117:4343-4354.

$84 \mathrm{Wu} \mathrm{G}, \mathrm{Gu}$ Y, Li S, et al. A genome-wide analysis of Arabidopsis Rop-interactive CRIB motif-containing proteins that act as Rop GTPase targets. Plant Cell 2001; 13:2841-2856.

85 Hwang JU, Gu Y, Lee YJ, et al. Oscillatory ROP GTPase activation leads the oscillatory polarized growth of pollen tubes. Mol Biol Cell 2005; 16:5385-5399.

86 Bokoch GM. Regulation of innate immunity by Rho GTPases. Trends Cell Biol 2005; 15:163-171.

87 Geiszt M, Leto TL. The Nox family of NAD[P]H oxidases: host defense and beyond. J Biol Chem 2004; 279:51715-51718.

88 Lapouge K, Smith SJ, Walker PA, et al. Structure of the TPR domain of p67phox in complex with Rac.GTP. Mol Cell 2000; 6:899-907.

89 Diebold BA, Bokoch GM. Molecular basis for Rac2 regulation of phagocyte NADPH oxidase. Nat Immunol 2001; 2:211215.

90 Groom QJ, Torres MA, Fordham-Skelton AP, et al. rbohA, a rice homologue of the mammalian gp91phox respiratory burst oxidase gene. Plant J 1996; 10:515-522.

91 Keller T, Damude HG, Werner D, et al. A plant homolog of the neutrophil NADPH oxidase gp91phox subunit gene encodes a plasma membrane protein with $\mathrm{Ca} 2+$ binding motifs. Plant Cell 1998; 10:255-266.

92 Torres MA, Onouchi H, Hamada S, et al. Six Arabidopsis thaliana homologues of the human respiratory burst oxidase (gp91phox). Plant J 1998; 14:365-370.

93 Torres MA, Dangl JL. Functions of the respiratory burst oxidase in biotic interactions, abiotic stress and development. Curr Opin Plant Biol 2005; 8:397-403.

94 Banfi B, Tirone F, Durussel I, et al. Mechanism of Ca2+ activation of the NADPH oxidase 5 (NOX5). J Biol Chem 2004; 279:18583-18591.
95 Sagi M, Fluhr R. Superoxide production by plant homologues of the gp91(phox) NADPH oxidase. Modulation of activity by calcium and by tobacco mosaic virus infection. Plant Physiol 2001; 126:1281-1290.

96 Kawasaki T, Henmi K, Ono E, et al. The small GTP-binding protein rac is a regulator of cell death in plants. Proc Natl Acad Sci U S A 1999; 96:10922-10926.

97 Ono E, Wong HL, Kawasaki T, et al. Essential role of the small GTPase Rac in disease resistance of rice. Proc Natl Acad Sci U S A 2001; 98:759-764.

98 Suharsono U, Fujisawa Y, Kawasaki T, et al. The heterotrimeric $G$ protein alpha subunit acts upstream of the small GTPase Rac in disease resistance of rice. Proc Natl Acad Sci U S A 2002; 99:13307-13312.

99 Kawasaki T, Koita H, Nakatsubo T, et al. Cinnamoyl-CoA reductase, a key enzyme in lignin biosynthesis, is an effector of small GTPase Rac in defense signaling in rice. Proc Natl Acad Sci U S A 2006; 103:230-235.

100 Hong Z, Zhang Z, Olson JM, et al. A novel UDP-glucose transferase is part of the callose synthase complex and interacts with phragmoplastin at the forming cell plate. Plant Cell 2001; 13:769-779.

101 Qadota H, Python CP, Inoue SB, et al. Identification of yeast Rho1p GTPase as a regulatory subunit of 1,3-beta-glucan synthase. Science 1996; 272:279-281.

102 Rivero F, Somesh BP. Signal transduction pathways regulated by Rho GTPases in Dictyostelium. J Muscle Res Cell Motil 2002; 23:737-749.

103 Bapteste E, Brinkmann H, Lee JA, et al. The analysis of 100 genes supports the grouping of three highly divergent amoebae: Dictyostelium, Entamoeba, and Mastigamoeba. Proc Natl Acad Sci U S A 2002; 99:1414-1419.

104 Christensen TM, Vejlupkova Z, Sharma YK, et al. Conserved subgroups and developmental regulation in the monocot rop gene family. Plant Physiol 2003; 133:1791-1808.

105 Saitou N, Nei M. The neighbor-joining method: a new method for reconstructing phylogenetic trees. Mol Biol Evol. 1987; 4:406-425.

106 Page RD. TreeView: an application to display phylogenetic trees on personal computers. Comput Appl Biosci. 1996; 12:357358. 